\title{
Implementación del Reglamento Sanitario Internacional en Cuba: evaluación de capacidades básicas del sector salud en provincias seleccionadas
}

\author{
Ángela Gala, ${ }^{1}$ María Eugenia Toledo, ${ }^{1}$ Yanisnubia Arias, ${ }^{2}$ \\ Manuel Díaz González, ${ }^{1}$ Ángel Manuel Álvarez Valdez, ${ }^{3}$ Gonzalo Estévez, ${ }^{3}$ \\ Rolando Miyar Abreu ${ }^{4}$ y Gustavo Kourí Flores ${ }^{1 \dagger}$
}

Forma de citar

Gala A, Toledo ME, Arias Y, Díaz González M, Álvarez Valdez AM, Estévez G, et al. Implementación del Reglamento Sanitario Internacional en Cuba: evaluación de capacidades básicas del sector salud en provincias seleccionadas. Rev Panam Salud Publica. 2012;32(3):207-16.

RESUMEN Objetivo. Obtener información de línea base sobre el estado de las capacidades básicas del sector salud a nivel local, municipal y provincial, a fin de facilitar la identificación de prioridades y orientar las políticas públicas dirigidas a garantizar los requisitos y capacidades establecidos en el Anexo 1A del Reglamento Sanitario Internacional de 2005 (RSI-2005).

Métodos. Se realizó un estudio descriptivo de corte transversal mediante la aplicación de un instrumento de evaluación de capacidades básicas referidas a la autonomía legal e institucional, el proceso de vigilancia e investigación y la respuesta frente a emergencias sanitarias en 36 entidades involucradas en el control sanitario internacional de los niveles local, municipal y provincial en las provincias de La Habana, Cienfuegos y Santiago de Cuba.

Resultados. Los policlínicos y centros provinciales de higiene y epidemiología de las tres provincias contaban con más del $75 \%$ de las capacidades básicas requeridas. Doce de 36 unidades disponían del $50 \%$ del marco legal e institucional implementado. La vigilancia e investigación de rutina presentaron una disponibilidad variable, mientras que las entidades de La Habana contaron con más del $40 \%$ de capacidades básicas en el campo de la respuesta ante eventos.

Conclusiones. Las provincias evaluadas cuentan con capacidades básicas instaladas que permitirán la implementación del RSI-2005 según el plazo previsto por la Organización Mundial de la Salud. Es necesario establecer y desarrollar planes de acción eficaces para consolidar a la vigilancia como una actividad esencial de seguridad nacional e internacional en términos de salud pública.

Palabras clave Reglamento Sanitario Internacional; evaluación; salud mundial; cooperación internacional; Cuba.

1 Instituto de Medicina Tropical "Pedro Kourí", La Habana, Cuba. La correspondencia se debe dirigir a Ángela Gala González. Correo electrónico: angela@ipk.sld.cu

2 Escuela Latinoamericana de Medicina, La Habana, Cuba.
3 Dirección Nacional de Higiene, Epidemiología y Microbiología, Ministerio de Salud Pública, La Habana, Cuba.

4 Consultor en la Escuela Nacional de Salud Pública, Cuba; ex-consultor de la Organización Panamericana de la Salud/Organización Mundial de la Salud en Cuba.
El Reglamento Sanitario Internacional (RSI) es un documento jurídicamente vinculante para todos los Estados parte de la Organización Mundial de la Salud (OMS), dirigido a prevenir la propaga- 
ción internacional de enfermedades. En otras palabras, se trata de un código de conducta que responsabiliza a los Estados parte por las medidas que adopten - u omitan - para minimizar los riesgos de propagación que pudieran existir para otros países (1).

En 2005, en la 58 a Asamblea Mundial de la Salud, se aprobó la última versión actualizada del Reglamento Sanitario Internacional (RSI-2005) — tras un proceso de revisión que abarcó una decena de años-, el que no entraría en vigor sino hasta junio de 2007 (2). Sus objetivos son prevenir la propagación internacional de enfermedades y de otros riesgos agudos para la salud pública, monitorear y controlar su evolución y dar una respuesta proporcionada y acotada a dichos riesgos evitando interferencias innecesarias con el tráfico y el comercio internacionales (1). Los países acordaron un plazo de cinco años a partir de su entrada en vigencia para implementar los cambios necesarios que garantizaran su funcionamiento $(2,3)$. A grandes líneas, se requería de capacidades específicas que aseguraran la funcionalidad del RSI2005 no solo dentro de las fronteras nacionales, sino también en la proyección internacional de cada Estado parte (4-6). De estas capacidades dependen funciones esenciales que intervienen en la detección oportuna y la respuesta proporcionada frente a acontecimientos que pueden poner en peligro a la salud pública $(7,8)$.

El alcance del RSI-2005 es tan amplio que considera importante a cualquier evento o riesgo que pueda causar una crisis en materia de salud pública internacional $(1,9)$. Asimismo, por "evento" entiende la manifestación de una enfermedad o un suceso, cualquiera sea su origen o procedencia, que entrañe o pueda entrañar un daño importante para cualquier persona (1).

En el artículo 5 y el Anexo 1 del RSI2005 se requiere que los países evalúen la capacidad existente de sus estructuras nacionales, así como sus habilidades y recursos de respaldo para garantizar los requisitos mínimos de vigilancia y respuesta, en un plazo de dos años después de su entrada en vigor $(1,6$, 10). En conformidad con esa directriz, el Ministerio de Salud Pública de Cuba (MINSAP), con el apoyo de la OMS, realizó una evaluación de las capacidades básicas requeridas para la implementación del RSI-2005 entre mayo y noviem- bre de 2008. Esta evaluación fue implementada en tres provincias, cada una perteneciente a sendas regiones del país (occidental, central y oriental), las que fueron seleccionadas según la magnitud del intercambio internacional registrado. El propósito de esa evaluación fue obtener información de línea base sobre el estado de las capacidades básicas del sector salud a nivel local, municipal y provincial, con el fin de facilitar la identificación de prioridades y orientar las políticas públicas dirigidas a garantizar los requisitos y capacidades establecidos en el Anexo 1 del RSI-2005.

\section{MATERIALES Y MÉTODOS}

Se realizó un estudio descriptivo, de corte transversal, mediante la aplicación de un instrumento de evaluación de capacidades básicas para sitios vulnerables, específicamente diseñado y validado por el equipo de investigación a partir de las recomendaciones de la OMS (Anexo 1A).

Toda la investigación fue coordinada y supervisada por el Instituto de Medicina Tropical "Pedro Kourí", bajo la dirección del Programa Nacional de Control Sanitario Internacional (PNCSI) del MINSAP de Cuba, y contó con el apoyo técnico y logístico de la Organización Panamericana de la Salud (OPS). El equipo de investigación estuvo integrado por médicos especialistas en las áreas de higiene y epidemiología, y con amplia experiencia en el control sanitario internacional y la organización y administración de programas y servicios de salud pública. Se realizó un taller previo a la investigación para examinar las prioridades y los objetivos de evaluación, revisar los instrumentos de evaluación, capacitar al personal encargado de su aplicación y realizar diversas pruebas al protocolo de evaluación.

Los sitios diana fueron identificados con la asesoría de un grupo de expertos. Se eligió una muestra por conveniencia de distintos tipos de entidades posiblemente vinculadas al ingreso de enfermedades o daños a la salud pública, las acciones de vigilancia y detección que llevaban a cabo y la capacidad de responder coordinadamente frente a eventos que pusieran en peligro la salud pública a nivel nacional $\mathrm{y} / \mathrm{o}$ internacional en las provincias de La Habana, Cienfuegos y Santiago de Cuba.

A nivel local se evaluaron todos los centros de estudios para extranjeros exis- tentes en cada una de las provincias seleccionadas, las clínicas internacionales, los servicios de salud de instituciones de deporte y recreación, así como también algunos policlínicos elegidos por estar radicados en comunidades con alta afluencia de viajeros o por su cercanía con atracciones turísticas. A nivel municipal, fueron incluidas las Unidades Municipales de Higiene y Epidemiología (UMHE) de los municipios cabecera y, en el caso de La Habana, también las ubicadas en los municipios de Regla, Boyeros y Playa por la existencia de puntos de entrada tales como escuelas internacionales e importantes centros turísticos. Finalmente, a nivel provincial fueron evaluados los Programas Provinciales de Control Sanitario Internacional —que incluyen las redes provinciales para la vigilancia, notificación, investigación y coordinación de la respuesta- en los Centros Provinciales de Higiene y Epidemiología (CPHE).

\section{Operacionalización de la evaluación}

El instrumento diseñado para evaluar las capacidades básicas en los sitios vulnerables estuvo dirigido a tres campos fundamentales: el grado de autonomía y responsabilidad legal, el proceso de vigilancia e investigación -en particular, los servicios básicos de detección, investigación y notificación- y el proceso de coordinación de la respuesta ante eventos de salud pública de importancia nacional e internacional (ESPINI) (cuadro 1). En este último caso, el énfasis estuvo puesto en la capacidad de poner en práctica medidas de intervención urgentes, la comunicación social existente durante una crisis y la evaluación y readecuación en la etapa de recuperación.

La información complementaria se obtuvo mediante observación directa, entrevistas con los actores, la aplicación de un cuestionario para medir las distintas competencias (detectar, notificar, verificar, evaluar, investigar, responder y comunicar) y revisión documental de evidencias.

\section{Análisis}

Las variables cualitativas incluyeron las categorías "total", "parcial" y "ninguno", las que fueron ponderadas de la siguiente forma: (la respuesta) "total" otorga 1 punto a la variable, "parcial" otorga 0,5 y "ninguno" no otorga pun- 
CUADRO 1. Instrumento utilizado para evaluar las capacidades básicas de entidades del sector salud, con miras a la implementación del Reglamento Sanitario Internacional (RSI), Cuba, 2008

\begin{tabular}{|c|c|c|c|}
\hline Área & Capacidad & Indicador & Verificación \\
\hline \multirow[t]{3}{*}{$\begin{array}{l}\text { Autonomía y } \\
\text { responsabilidad } \\
\text { legal }\end{array}$} & \multirow[t]{3}{*}{$\begin{array}{l}\text { Legislación, } \\
\text { normas, } \\
\text { protocolos } \\
\text { de funciones } \\
\text { y acciones }\end{array}$} & $\begin{array}{l}\text { Porcentaje de } \\
\text { disponibilidad y uso } \\
\text { de instrumentos } \\
\text { legislativos }\end{array}$ & $\begin{array}{l}\text { Disponibilidad y conocimiento de leyes, } \\
\text { decretos-leyes, decretos, reglamentos y } \\
\text { resoluciones relacionadas con la salud pública }\end{array}$ \\
\hline & & $\begin{array}{l}\text { Porcentaje de } \\
\text { disponibilidad y } \\
\text { uso de protocolos } \\
\text { y fichas de } \\
\text { procedimientos }\end{array}$ & $\begin{array}{l}\text { Protocolos de trabajo, fichas técnicas de } \\
\text { procedimientos en puestos de trabajo }\end{array}$ \\
\hline & & $\begin{array}{l}\text { Trazabilidad de la } \\
\text { organización de } \\
\text { funciones en todos } \\
\text { los niveles de la } \\
\text { entidad }\end{array}$ & $\begin{array}{l}\text { Roles, responsabilidades, flujograma } \\
\text { organizacional }\end{array}$ \\
\hline \multirow[t]{6}{*}{$\begin{array}{l}\text { Vigilancia e } \\
\text { investigación }\end{array}$} & Estructura & $\begin{array}{l}\text { Cobertura de recursos } \\
\text { humanos } \\
\text { Cobertura de recursos } \\
\text { físicos } \\
\text { Disponibilidad } \\
\text { de recursos } \\
\text { financieros }\end{array}$ & $\begin{array}{l}\text { Plantilla, formación profesional, cobertura de } \\
\text { servicios } \\
\text { Infraestructura, equipamiento, recursos } \\
\text { materiales, transporte, tecnología } \\
\text { Mediante la existencia de una línea de } \\
\text { presupuesto para vigilancia de eventos de } \\
\text { riesgo, vías de movilización }\end{array}$ \\
\hline & \multirow[t]{5}{*}{ Proceso } & $\begin{array}{l}\text { Cumplimiento de la } \\
\text { vigilancia }\end{array}$ & $\begin{array}{l}\text { Servicios de vigilancia en atención primaria } \\
\text { de salud, sistema de detección, fuentes de } \\
\text { información, notificación, investigación de } \\
\text { brotes, manejo del Anexo } 2 \text { del RSI }\end{array}$ \\
\hline & & $\begin{array}{l}\text { Uso del análisis } \\
\text { de datos para la } \\
\text { detección }\end{array}$ & $\begin{array}{l}\text { Sistema de análisis de datos, uso } \\
\text { de tecnología, trazabilidad, análisis } \\
\text { de riesgos }\end{array}$ \\
\hline & & $\begin{array}{l}\text { Uso de notificación, } \\
\text { verificación y } \\
\text { evaluación }\end{array}$ & $\begin{array}{l}\text { Funcionamiento del sistema de notificación, } \\
\text { flujograma, vías, coordinación, cobertura } 24 \text { h/7 } \\
\text { días, retroalimentación }\end{array}$ \\
\hline & & $\begin{array}{l}\text { Aplicación de la } \\
\text { investigación }\end{array}$ & $\begin{array}{l}\text { Protocolos de investigación, uso de formularios } \\
\text { por evento, validación de las acciones de } \\
\text { control, controles de calidad, actualización. }\end{array}$ \\
\hline & & $\begin{array}{l}\text { Nivel de ejecución de } \\
\text { la comunicación }\end{array}$ & $\begin{array}{l}\text { Flujo de información entre niveles del Sistema } \\
\text { Nacional de Salud, medios de comunicación, } \\
\text { comunicación interna, comunicación } \\
\text { a prestadores, comunicación pública, } \\
\text { comunicación con enfoque de riesgo }\end{array}$ \\
\hline \multirow[t]{6}{*}{ Respuesta } & \multirow[t]{3}{*}{ Estructura } & $\begin{array}{l}\text { Cobertura de recursos } \\
\text { humanos (Grupo } \\
\text { de respuesta } \\
\text { rápida) }\end{array}$ & $\begin{array}{l}\text { Brigada de respuesta rápida, disponibilidad } \\
24 \mathrm{~h} / 7 \text { días, capacitación (programa de } \\
\text { capacitación permanente, preparación) }\end{array}$ \\
\hline & & $\begin{array}{l}\text { Cobertura de recursos } \\
\text { físicos }\end{array}$ & $\begin{array}{l}\text { Infraestructura de trabajo, equipamiento, } \\
\text { tecnología, Equipos de Protección Personal } \\
\text { (EPP), reservas para tiempo de emergencia, } \\
\text { facilidades de transporte, estructuras de } \\
\text { control, unidades de referencia. }\end{array}$ \\
\hline & & $\begin{array}{l}\text { Disponibilidad } \\
\text { de recursos } \\
\text { financieros }\end{array}$ & $\begin{array}{l}\text { Recursos financieros de reserva, capacidad } \\
\text { de movilización de recursos, algoritmo de } \\
\text { movilización, capacitación, informes de } \\
\text { ejecución financiera }\end{array}$ \\
\hline & \multirow[t]{3}{*}{ Coordinación } & $\begin{array}{l}\text { Aplicación de los } \\
\text { protocolos de } \\
\text { acción y planes } \\
\text { de emergencia }\end{array}$ & $\begin{array}{l}\text { Protocolos de acción, plan de respuesta de los } \\
\text { servicios de emergencias, guías de reservas } \\
\text { estratégicas, guías y manuales de movilización } \\
\text { de recursos ensayados y actualizados }\end{array}$ \\
\hline & & $\begin{array}{l}\text { Ejecución de la } \\
\text { coordinación. } \\
\text { Empleo de la } \\
\text { comunicación } \\
\text { de riesgo }\end{array}$ & $\begin{array}{l}\text { Coordinación con áreas estratégicas dentro y } \\
\text { fuera del sector salud, acciones de respuesta } \\
\text { por responsabilidad y área de competencia, } \\
\text { evaluación periódica sobre el impacto de las } \\
\text { medidas }\end{array}$ \\
\hline & & $\begin{array}{l}\text { Disponibilidad } \\
\text { y preparación } \\
\text { de los servicios } \\
\text { de respuesta } \\
\text { emergente }\end{array}$ & $\begin{array}{l}\text { Coordinación local de área de cuarentena, } \\
\text { servicios en centros de referencia } \\
\text { especializados, transportación sanitaria en caso } \\
\text { de eventos de salud pública de importancia } \\
\text { nacional e internacional (ESPINI) }\end{array}$ \\
\hline
\end{tabular}

Fuente: elaboración de los autores. tos. Luego, los valores de las diferentes variables de cada indicador fueron sumados para obtener la media aritmética de cada grupo de observaciones (valor final del indicador). Los puntos de corte establecidos para la calificación cualitativa de las capacidades fueron: $30 \%$ o menos de disponibilidad (se consideró "no existente"), de 31\% a 69\% ("fortalecer capacidad" [están disponibles pero no satisfacen su función según los requisitos del RSI-2005]) y 70\% o más ("capacidad funcional" [están disponibles y cumplen todos los requisitos del RSI]). Por último, al analizar el estado global de cada capacidad con objeto de cuantificar su grado de disponibilidad, se sumaron los valores de las categorías "capacidad funcional" y "fortalecer capacidad".

\section{RESULTADOS}

Se evaluaron 36 unidades de servicios de salud pública, 14 en La Habana y Santiago de Cuba respectivamente, y 8 en Cienfuegos. Del total de 28 entidades evaluadas a nivel local, 13 correspondieron a centros de estudios con matrícula internacional, 9 a policlínicos, 4 a clínicas internacionales y 2 a instituciones deportivas. Asimismo, también se evaluaron cinco UMHE y, en las provincias seleccionadas, los CPHE con sus laboratorios de referencia (cuadro 2).

En el cuadro 3 se muestran los promedios globales obtenidos para las capacidades básicas evaluadas en cada provincia. En La Habana, los centros de estudios, los policlínicos, la clínica internacional y el CPHE superaron el 70\% de disponibilidad de las capacidades básicas requeridas. De esas capacidades, se calificaron como funcionales $40 \%$ en la clínica internacional, $24 \%$ en los centros de estudios y $20 \%$ en el CPHE. Las tres UMHE promediaron $46,7 \%$ de disponibilidad.

En Santiago de Cuba, los policlínicos, el CPHE y la UMHE disponían de más de $70 \%$ de las capacidades básicas requeridas, de las cuales $10 \%, 20 \%$ y $26,7 \%$ respectivamente, fueron calificadas como funcionales. Las clínicas internacionales promediaron $63,3 \%$ de disponibilidad de capacidades básicas y solo 6,67\% eran funcionales. Los centros de estudios y de deportes contaron con $46,7 \%$ de las capacidades que debían ser fortalecidas ("a fortalecer").

La provincia de Cienfuegos tuvo el mayor porcentaje de capacidades fun- 
CUADRO 2. Tipos de entidades estudiadas para evaluar capacidades básicas de salud con miras a la implementación del Reglamento Sanitario Internacional, por provincia y nivel de gobierno, Cuba, 2008

\begin{tabular}{|c|c|c|c|c|c|c|c|c|c|}
\hline \multirow[b]{3}{*}{ Nivel } & \multirow[b]{3}{*}{ Tipo de entidad } & \multicolumn{6}{|c|}{ Provincia } & & \\
\hline & & \multicolumn{2}{|c|}{ La Habana } & \multicolumn{2}{|c|}{ Santiago de Cuba } & \multicolumn{2}{|c|}{ Cienfuegos } & \multicolumn{2}{|c|}{ Total } \\
\hline & & No. & $\%$ & No. & $\%$ & No. & $\%$ & No. & $\%$ \\
\hline \multirow[t]{4}{*}{ Local } & Centros de estudios & 5 & 38,46 & 5 & 38,46 & 3 & 23,08 & 13 & 35,14 \\
\hline & Instituciones deportivas & 1 & 50,00 & 1 & 50,00 & 0 & 0,00 & 2 & 5,41 \\
\hline & Clínicas internacionales & 1 & 25,00 & 2 & 50,00 & 1 & 25,00 & 4 & 10,81 \\
\hline & Policlínicos & 3 & 30,00 & 4 & 50,00 & 2 & 20,00 & 9 & 27,03 \\
\hline Municipal & UMHE & 3 & 60,00 & 1 & 20,00 & 1 & 20,00 & 5 & 13,51 \\
\hline Provincial & CPHE & 1 & 33,33 & 1 & 33,33 & 1 & 33,33 & 3 & 8,11 \\
\hline Total & & 14 & 38,89 & 14 & 38,89 & 8 & 22,22 & 36 & 100,00 \\
\hline
\end{tabular}

Fuente: elaboración de los autores.

Nota: UMHE: Unidad Municipal de Higiene y Epidemiología; CPHE: Centro Provincial de Higiene y Epidemiología.

CUADRO 3. Promedio global de las capacidades básicas de salud evaluadas con miras a la implementación del Reglamento Sanitario Internacional, por tipo de entidad y provincia, Cuba, 2008

\begin{tabular}{llrrrr}
\hline & \multicolumn{1}{c}{$\begin{array}{c}\text { Tipo } \\
\text { Provincia }\end{array}$} & \multicolumn{4}{c}{ Capacidad } \\
\cline { 3 - 6 } & \multicolumn{1}{c}{ de entidad } & Funcional & $\begin{array}{c}\text { Requiere } \\
\text { fortalecimiento }\end{array}$ & $\begin{array}{c}\text { No } \\
\text { existente }\end{array}$ & $\begin{array}{c}\text { Total } \\
\text { disponible }\end{array}$ \\
\hline La Habana & Centro de estudios & 24,00 & 46,70 & 25,30 & 70,70 \\
& Instituciones deportivas & 0,00 & 20,00 & 80,00 & 20,00 \\
& Clínica internacional & 40,00 & 53,33 & 6,67 & 93,33 \\
& Policlínicos & 6,67 & 73,30 & 20,00 & 80,00 \\
Santiago de Cuba & 0,00 & 46,70 & 53,30 & 46,70 \\
& UMHE & 20,00 & 80,00 & 0,00 & 100,00 \\
& CPHE & 2,70 & 44,00 & 53,30 & 46,70 \\
& Centro de estudios & 0,00 & 46,70 & 53,30 & 46,70 \\
& Instituciones deportivas & 6,67 & 53,33 & 36,70 & 63,30 \\
& Clínica internacional & 10,00 & 78,33 & 11,70 & 88,30 \\
& Policlínicos & 26,67 & 46,67 & 20,00 & 73,30 \\
& UMHE & 20,00 & 66,67 & 13,30 & 86,70 \\
& CPHE & 4,44 & 37,78 & 57,80 & 42,20 \\
& Centro de estudios & 0,00 & 33,33 & 66,70 & 33,30 \\
& Clínica internacional & 43,33 & 40,00 & 16,70 & 83,30 \\
& Policlínicos & 46,67 & 26,67 & 26,67 & 73,30 \\
& UMHE & 46,67 & 40,00 & 13,30 & 86,70
\end{tabular}

Fuente: elaboración de los autores.

Nota: UMHE: Unidad Municipal de Higiene y Epidemiología; CPHE: Centro Provincial de Higiene y Epidemiología.

a Sumatoria de las capacidades funcionales y las que requieren fortalecimiento.

cionales en policlínicos (43,3\%), UMHE y CPHE $(46,7 \%)$, todas entidades que sobrepasaron el $70 \%$ de disponibilidad. Los centros de estudios y la clínica internacional, por su parte, solo alcanzaron $42,2 \%$ y $33,3 \%$ respectivamente de disponibilidad de capacidades básicas requeridas.

\section{Autonomía legal e institucional}

En cuanto al grado de autonomía legal e institucional necesario para la implementación del RSI-2005, de un total de nueve policlínicos evaluados, ocho alcanzaron un grado de cumplimiento de entre $35 \%$ y $50 \%$. Solo las clínicas internacionales radicadas en Santiago de Cuba contaron con un porcentaje de 50\%. Las UMHE de Santiago de Cuba y Cienfuegos alcanzaron un nivel de cumplimiento de $70 \%$, pero ninguna de las evaluadas en La Habana disponía de este campo. Los CPHE de Santiago de Cuba y Cienfuegos alcanzaron más de $60 \%$ de disponibilidad $\mathrm{y}$, el de La Habana, un 50\% (figura 1).

\section{Vigilancia e investigación de rutina}

Dentro de este grupo se evaluaron las capacidades estructurales, incluidos infraestructura, tecnología, recursos humanos y materiales de soporte, así como los procesos asociados a la detección de eventos, su notificación, investigación, el estudio de los riesgos y la comunicación de rutina. Según se observa en la figura 2, de los centros de estudios evaluados, solo "Cojímar" y la Escuela Latinoamericana de Medicina "Mártires de Girón" de La Habana registraron un cumpli- miento de $70 \%$ en este campo; Tarará registró $60 \%$. El porcentaje sobrepasó el $70 \%$ en los policlínicos de Cienfuegos y alcanzó un $50 \%$ en todos los de La Habana y Santiago de Cuba. Los CPHE presentaron un grado de cumplimiento de $60 \%$ en La Habana y Santiago de Cuba, mientras que solo la clínica internacional "Cira García" alcanzó un 80\% — las dos de Santiago de Cuba informaron $40 \%$.

\section{Respuesta durante emergencias de salud pública}

Fundamentalmente se evaluó la disponibilidad de planes para enfrentar emergencias de salud pública - contemplando la organización de los recursos humanos y materiales-, la reorganización de los servicios y la comunicación social de riesgo. En la figura 3, se observa que solo cuatro de 15 centros de estudios e instituciones deportivas disponían de más de $50 \%$ de las capacidades implementadas, de los cuales dos sobrepasaban el $75 \%$. En siete de nueve policlínicos se alcanzó un 50\%, destacándose los policlínicos área II de Cienfuegos y Julián Grimau de Santiago de Cuba con un porcentaje superior a 70\%. Los CPHE mostraron una mayor variabilidad en el cumplimiento, presentando $60 \%$ el de La Habana y $40 \%$ el de Santiago de Cuba. Las UMHE de Cienfuegos y del municipio Playa de La Habana informaron un $50 \%$, mientras que solo la clínica "Cira García" alcanzó el 60\%.

En la figura 4 se puede observar una exploración de los promedios globales de las capacidades básicas correspondientes a los grupos Vigilancia e investigación de rutina y Respuesta durante ESPINI en policlínicos provinciales. En cuanto a la vigilancia e investigación de rutina, se observó una situación favorable en las 
FIGURA 1. Promedio global del marco legal e institucional evaluado con miras a la implementación del Reglamento Sanitario Internacional, Cuba, 2008

Centros de estudios e instituciones deportivas

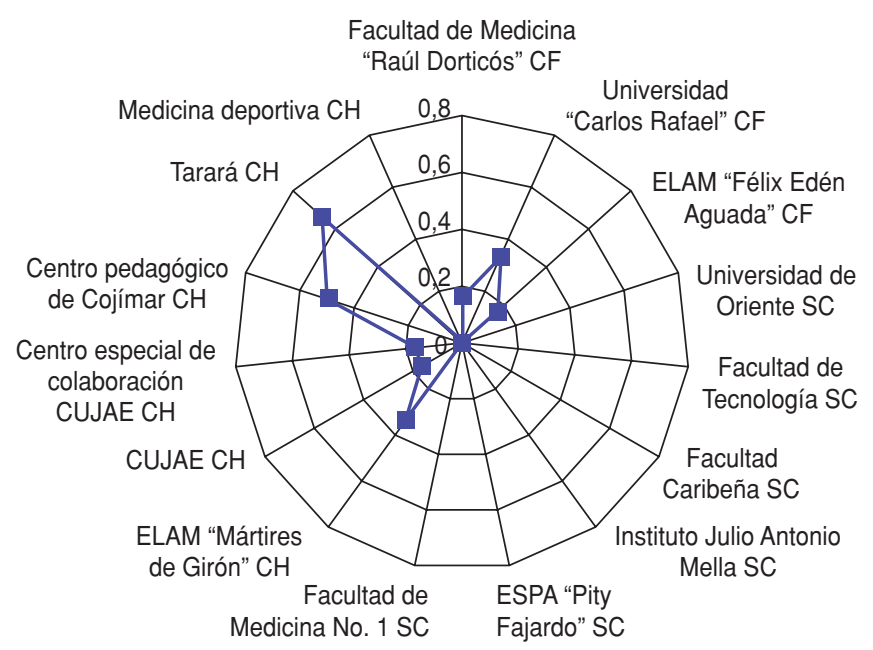

Unidades municipales y centros provinciales de higiene y epidemiología

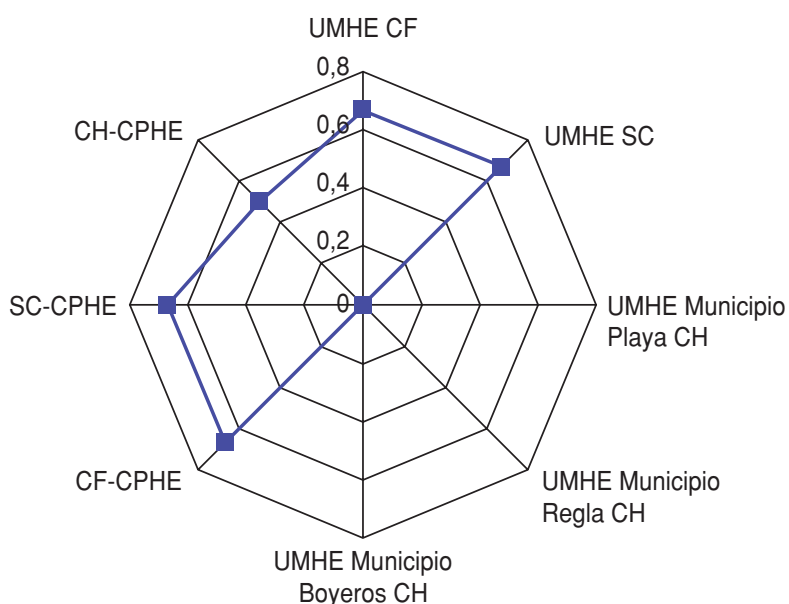

Policlínicos
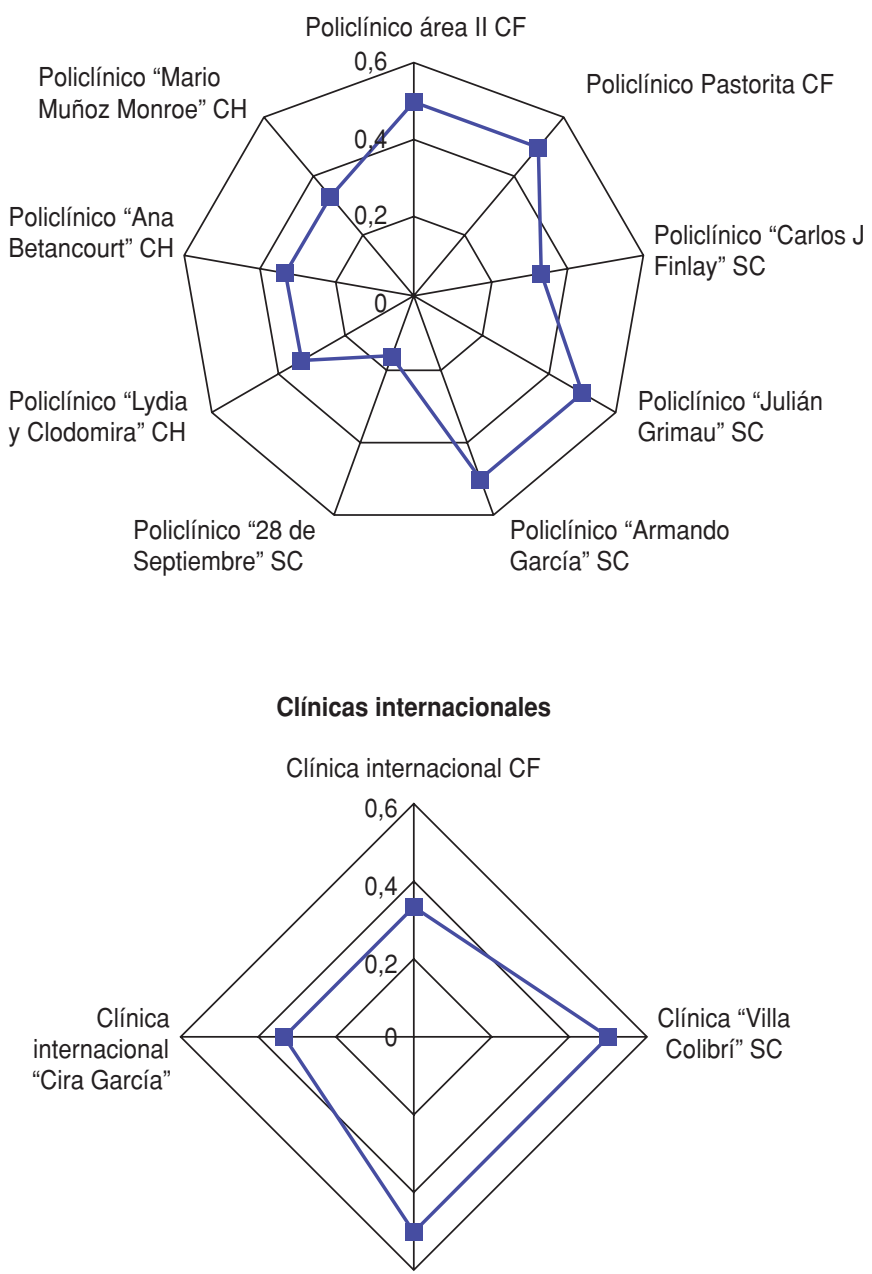

Clínica internacional SC

Fuente: elaboración de los autores.

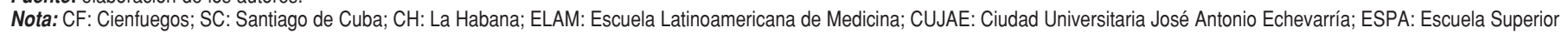
de Perfeccionamiento Atlético; UMHE: Unidades municipales de higiene y epidemiología; CPHE: Centros provinciales de higiene y epidemiología.

provincias ya que todas contaban con capacidades implementadas parcialmente, sobresaliendo la de Cienfuegos con la mayoría (4/7) de las capacidades listas para cumplir los requisitos del RSI-2005.

La respuesta ante ESPINI reveló una situación diferente, donde La Habana estuvo en ventaja sobre el resto ya que sus policlínicos contaban con planes de emergencia y protocolos de acción, así como con el resto de las capacidades implementadas parcialmente. Los policlínicos de Santiago de Cuba, que presentaron una total disponibilidad de capacidad de coordinación de la respuesta, no disponían en cambio de planes para enfrentar emergencias de salud pública. Por su parte, la provincia de Cienfuegos contaba tanto con recursos físicos y financieros como con planes de respuesta por parte de los servicios de salud.

\section{DISCUSIÓN}

La evaluación de las capacidades básicas de los Estados parte - según lo establecido en el propio reglamento- es medular para el proceso de implementación del RSI-2005, el cual es jurídicamente vinculante para todos los países firmantes (1). El consiguiente diagnóstico permitirá desarrollar planes de acción eficaces para solucionar las dificultades encontradas, reduciendo así los riesgos derivados del tráfico internacional de personas y bienes. Hoy por hoy, la situación a la que se enfrenta la salud pública mundial ha determinado la necesidad de acelerar y estandarizar los procesos de diagnóstico, con el propósito de dar una respuesta adecuada a los requerimientos establecidos por el RSI-2005 (1, 11, 12).

Para implementar estas nuevas exigencias del RSI-2005, con vistas a reducir los riesgos de propagación de enfermedades a través del tráfico internacional $-\mathrm{y} \sin$ interferencias innecesarias-, será necesario crear nuevas redes de 
FIGURA 2. Promedio global de las capacidades básicas de vigilancia e investigación de rutina evaluadas con miras a la implementación del Reglamento Sanitario Internacional, Cuba, 2008

Centros de estudios e instituciones deportivas

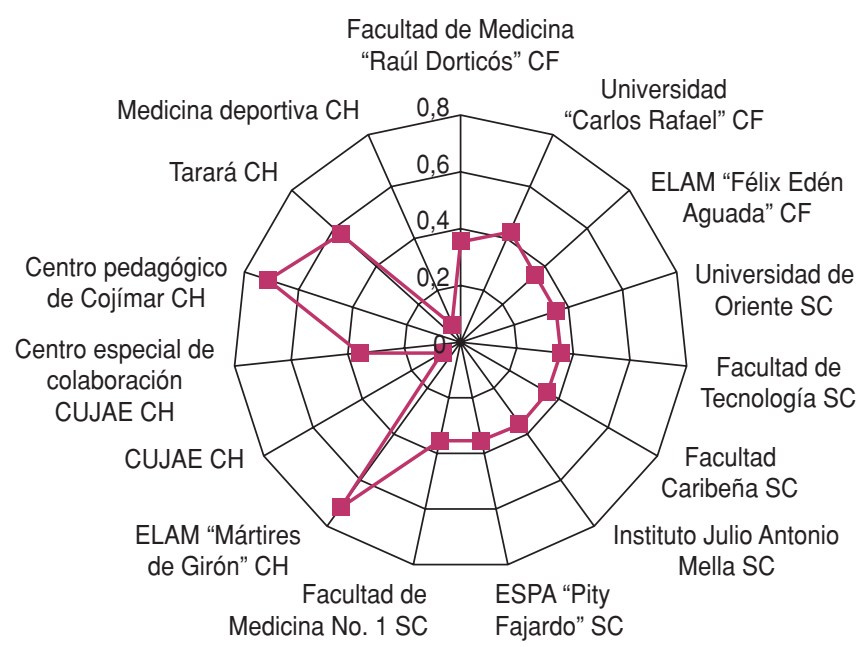

Unidades municipales y centros provinciales de higiene y epidemiología

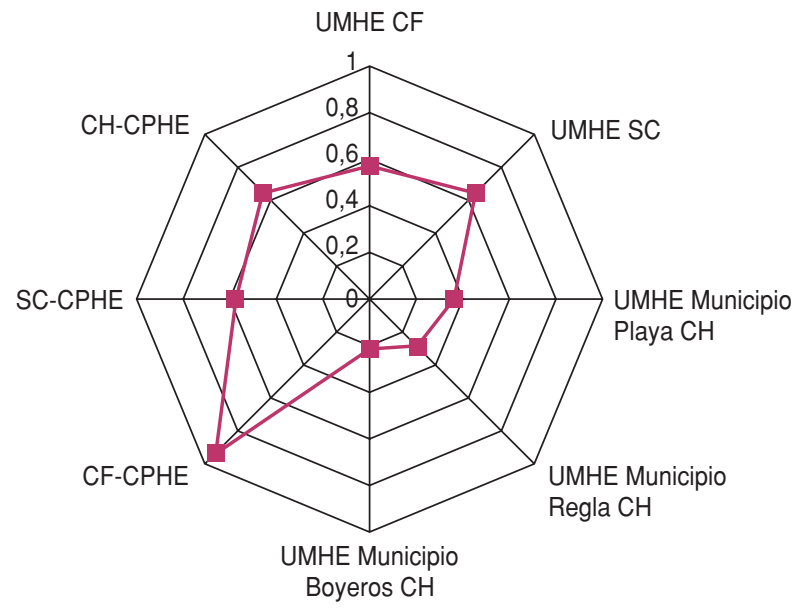

Policlínicos

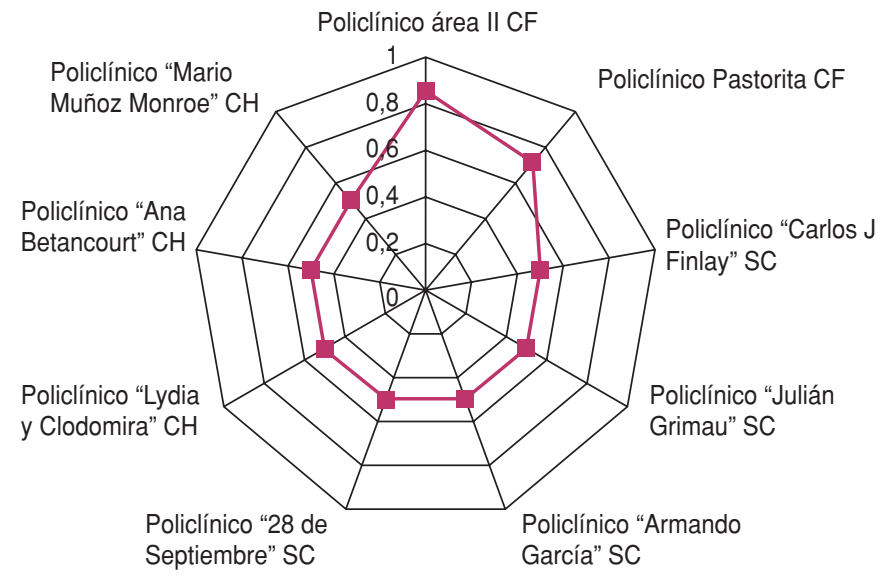

Clínicas internacionales

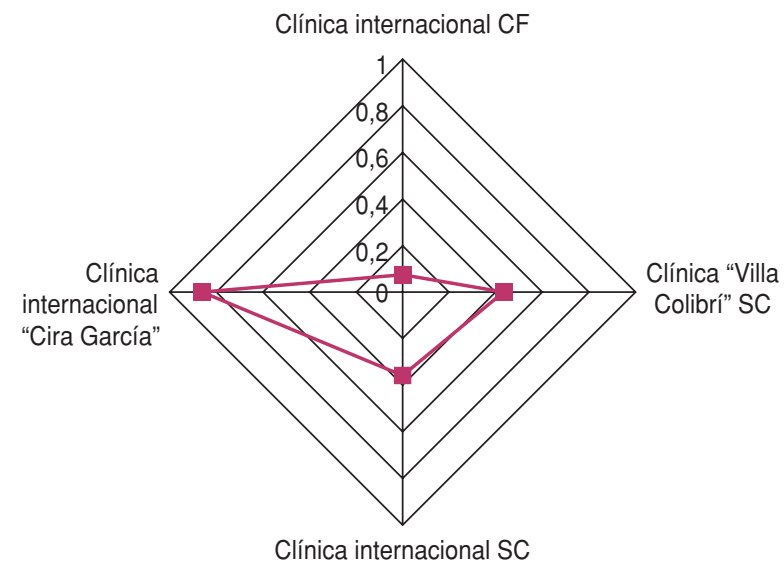

Clínica internacional SC

Fuente: elaboración de los autores.

Nota: CF: Cienfuegos; SC: Santiago de Cuba; CH: La Habana; ELAM: Escuela Latinoamericana de Medicina; CUJAE: Ciudad Universitaria José Antonio Echevarría; ESPA: Escuela Superior de Perfeccionamiento Atlético; UMHE: Unidades municipales de higiene y epidemiología; CPHE: Centros provinciales de higiene y epidemiología.

vigilancia, y fortalecer las existentes, así como realizar planes de capacitación continua actualizados para el personal vinculado con la seguridad de la salud internacional $(1,3,4,6,12,13) .^{5}$

En el presente estudio, donde se utilizó un instrumento específico para evaluar las capacidades básicas en servicios de salud pública, se lograron obtener valores de implementación que sirvie-

\footnotetext{
5 En este sentido, se puede decir que la pandemia de influenza $\mathrm{A}(\mathrm{H} 1 \mathrm{N1})$ en 2009, fue el primer reto enfrentado por el mundo con el nuevo RSI-2005 vigente (14).
}

ron de línea de base para trabajar en el desarrollo y fortalecimiento de las redes de detección, evaluación, notificación y respuesta de las provincias cubanas de referencia. Los resultados obtenidos mediante dicho instrumento en 2008, antes del plazo estipulado por la OMS, muestran que en términos globales las provincias evaluadas cuentan con un grado aceptable de capacidades básicas instaladas, si bien se identificaron áreas sensibles que requieren un tratamiento prioritario para planificar la implementación en todo en el país.

\section{Autonomía y responsabilidad legal}

Dentro de los requisitos que el RSI2005 dispone para los Estados parte no se incluye el de que necesariamente revisen su legislación interna, siempre y cuando cumplan con las obligaciones asumidas. No obstante, es imprescindible que cuenten con un marco jurídico adecuado para sustentar y hacer posibles todas las acciones previstas $(1,15,16)$.

La ausencia de inventarios e instrumentos legales de consulta por parte de 
FIGURA 3. Promedio global de las capacidades básicas de respuesta frente a eventos de salud pública de importancia nacional e internacional (ESPINI), con miras a la implementación del Reglamento Sanitario Internacional, Cuba, 2008

Centros de estudios e instituciones deportivas

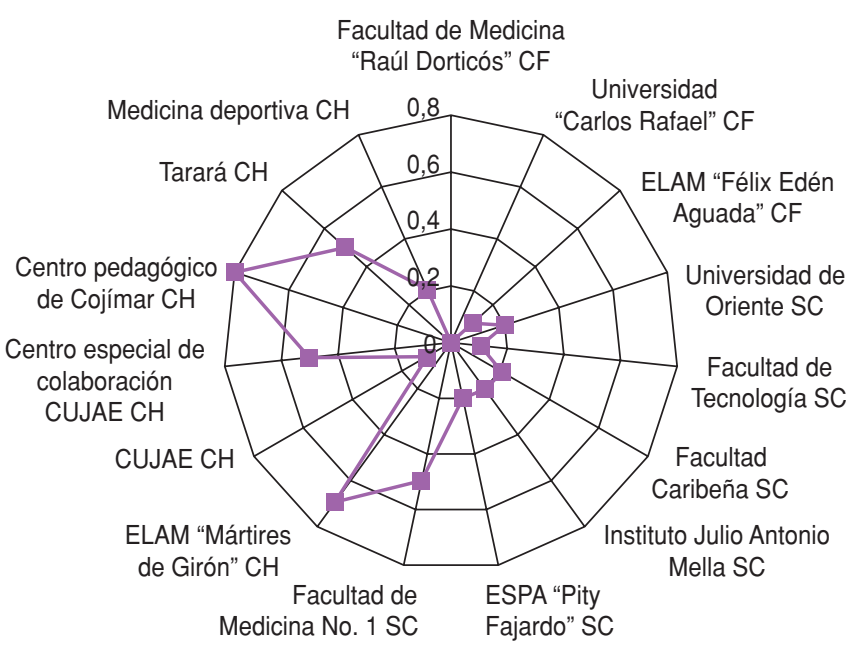

Unidades municipales y centros provinciales de higiene y epidemiología

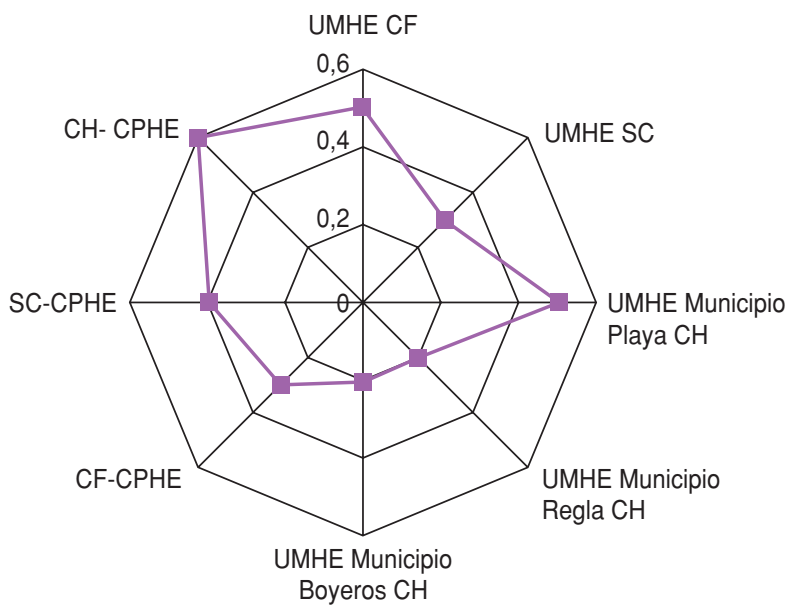

Policlínicos

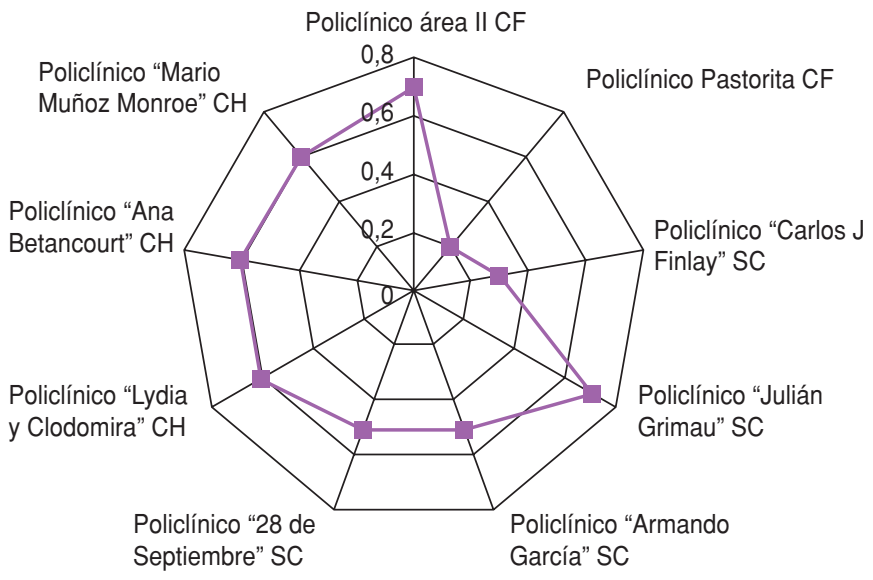

Fuente: elaboración de los autores.

Nota: CF: Cienfuegos; SC: Santiago de Cuba; CH: La Habana; ELAM: Escuela Latinoamericana de Medicina; CUJAE: Ciudad Universitaria José Antonio Echevarría; ESPA: Escuela Superior de Perfeccionamiento Atlético; UMHE: Unidades municipales de higiene y epidemiología; CPHE: Centros provinciales de higiene y epidemiología.

los profesionales involucrados predominó en la mayoría de las entidades evaluadas, salvo en el nivel provincial. Una mención especial merecen los centros de estudios y deportivos, que cuentan con servicios de salud pública pero sin el vínculo legal y administrativo necesario para favorecer el trabajo integrado de las partes. En todas las entidades se observó la falta de protocolos, procesos y procedimientos de actuación que garanticen la continuidad de los servicios, especialmente en los centros de estudios e instituciones deportivas, donde la mayoría (13 de 15) no disponían del marco legal ni tenían organizadas sus funciones de forma apropiada.

En este sentido, Cuba ha emprendido un proceso de identificación de la legislación nacional pertinente, mientras que otros países de la región han debido revisar o enmendar sus ordenamientos jurídicos para poder reconocer e incluir al RSI-2005 (11, 13, 17, 18). La actualización del marco legislativo cubano forma parte de la política de fortalecimiento del Sistema Nacional de Salud Pública, y está fundamentalmente dirigida a alcanzar mayores índices de eficiencia y efectividad en las acciones de salud, mejorando el nivel general de calidad de los servicios y preservando los logros alcanzados (19).

\section{Vigilancia e investigación de rutina}

El artículo 5 del RSI-2005 compromete a los países a desarrollar y mantener capacidades de detección, verificación, notificación, investigación y reporte de eventos de salud pública con potencial de generar crisis a la salud pública (1). En Cuba, la atención primaria de la salud, basada en las estrategias del médico y la enfermera de la familia, y el acceso universal y gratuito a la atención sanitaria, ofrecen 
FIGURA 4. Promedio global de capacidades básicas de vigilancia e investigación de rutina y de respuesta frente a eventos de salud pública de importancia nacional e internacional (ESPINI), con miras a la implementación del Reglamento Sanitario Internacional, policlínicos de provincias seleccionadas, Cuba, 2008

\section{CAPACIDADES DE VIGILANCIA E INVESTIGACIÓN DE RUTINA}

La Habana

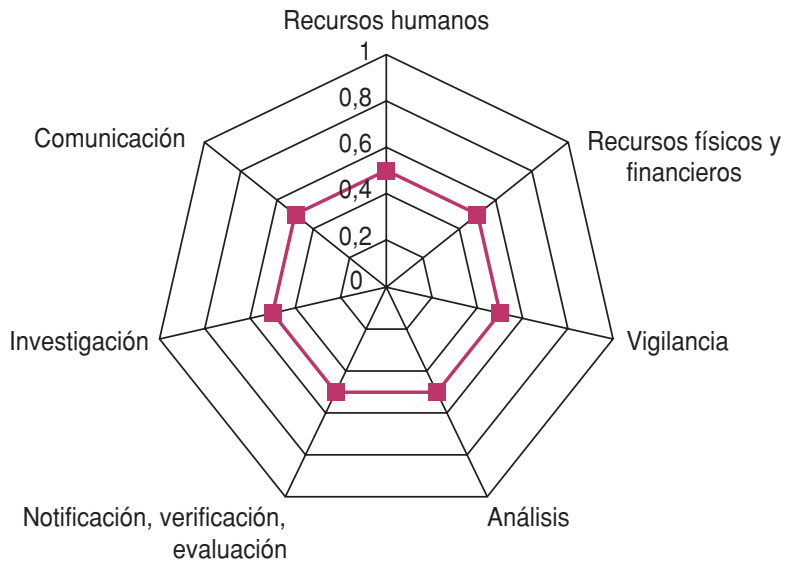

Santiago de Cuba

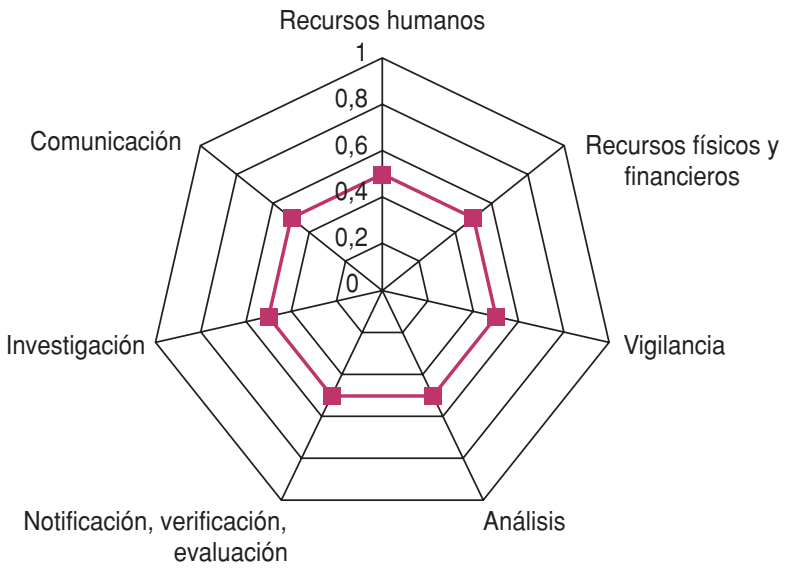

Cienfuegos

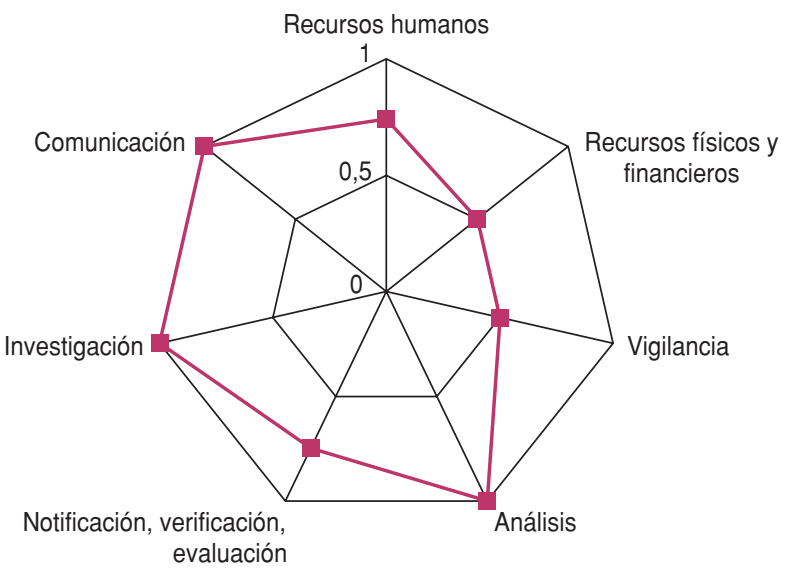

Fuente: elaboración de los autores.

Nota: Categorías. Capacidad total: 1 ; Capacidad parcial: 0,$5 ;$ Sin capacidad: 0 .

\section{CAPACIDADES DE RESPUESTA FRENTE A ESPINI}

La Habana

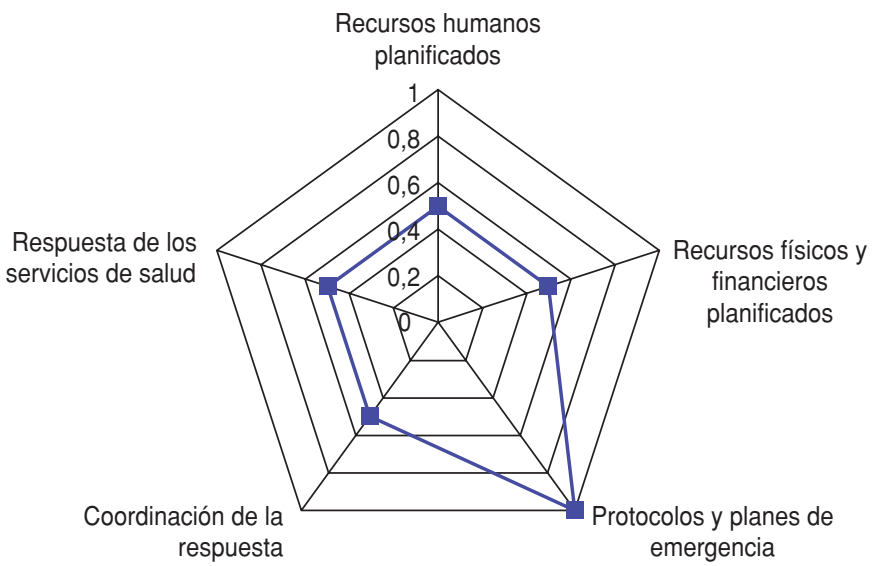

Santiago de Cuba

Recursos humanos planificados

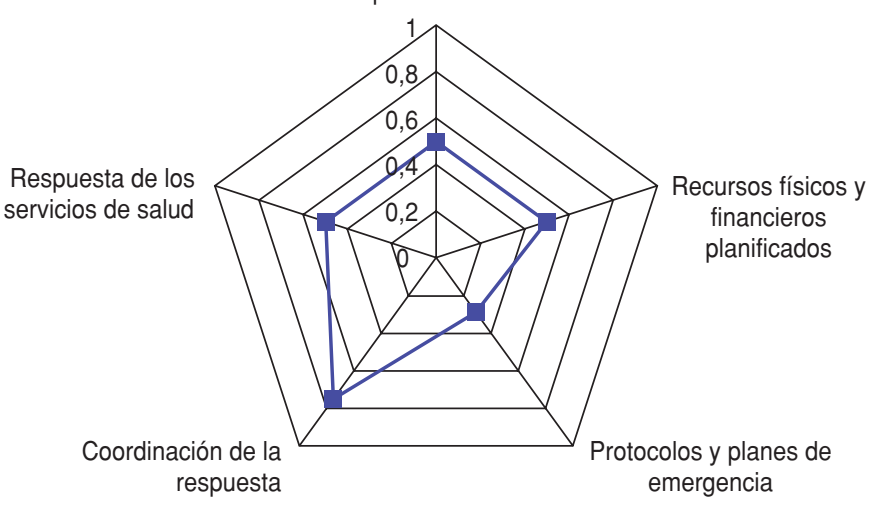

Cienfuegos

Recursos humanos planificados

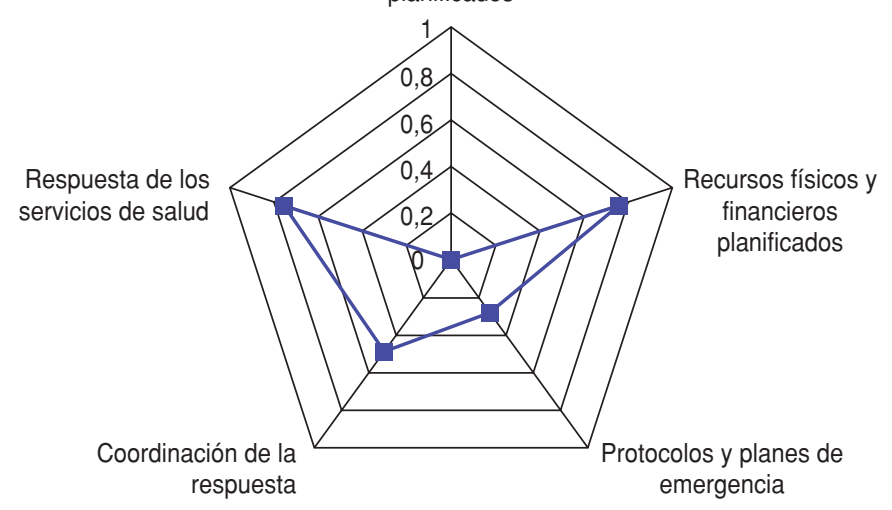


un contexto favorable para emprender la implementación del RSI-2005. Las principales dificultades halladas se enmarcaron en la organización de los servicios y la disponibilidad de guías y herramientas de vigilancia, especialmente en las entidades de los niveles local y municipal.

En 2009, con la emergencia de la influenza $\mathrm{A}(\mathrm{H} 1 \mathrm{~N} 1)$, se inició de inmediato la preparación del sistema para enfrentar su posible propagación en el territorio nacional (20). Los resultados de la presente evaluación sirvieron de sustrato para la priorización de acciones dirigidas al fortalecimiento de la vigilancia en todos los niveles.

El indicador que describe la capacidad básica referida a los recursos humanos se encontró disponible parcialmente en todas las entidades. Se trata de un indicador compuesto que combina aspectos relativos a la cobertura, el número previsto de personal técnico en las funciones evaluadas y el dominio de los procesos de vigilancia, investigación y comunicación de eventos. Si bien la cobertura resultó suficiente en las entidades evaluadas - con algunas pocas excepciones-, pudieron identificarse brechas significativas en el conocimiento de los eventos con riesgo para la salud pública y los enfoques para su manejo y control.

Al momento de realizarse el presente estudio, la respuesta frente a emergencias epidémicas se sustentaba sobre la base de planes de contingencia dirigidos específicamente a las enfermedades prioritarias en Cuba, como el dengue, el paludismo, las meningitis bacterianas $\mathrm{O}$ virales y otras de importancia por su carácter epidémico. Los recursos humanos disponibles para enfrentar emergencias de este tipo eran los mismos que realizaban las acciones de rutina y no se contaba con una capacitación formal sobre emergencias de salud pública y el modo de manejarlas. Las brigadas de respuesta rápida no existían en todas las entidades $\mathrm{y}$, donde lo hacían, no estaban protocolizadas o no tenían una coordinación planificada y probada en cuanto a su funcionalidad. Tampoco se hallaba protocolizada la movilización de los recursos humanos, la cual cuando se realizaba en tiempos de crisis redundaba en una respuesta inadecuada para el riesgo. Los planes de acción carecían de actualización, no se difundían entre las partes interesadas ni eran probados mediante ejercicios de simulación. Otra carencia fue la falta de una estrategia de comunicación para tiempos de crisis, si bien es frecuente el uso de murales y pancartas para la promoción de medidas sanitarias.

La vigilancia epidemiológica cubana es resuelta fundamentalmente por el médico de familia en la comunidad, pero su coordinación, uso, evaluación y actualización se realiza en el nivel intermedio (provincial), donde los responsables - los directivos de los programas de controlno siempre pueden asistir a cursos de capacitación o de actualización debido probablemente a la gran carga operacional que demandan sus tareas de rutina. Una política de cuadros adecuada, que incluya la capacitación sostenible y continua del personal, aseguraría la formación y actualización de competencias en consonancia con el desarrollo de las ciencias y el conocimiento de los problemas de salud que afectan a los seres humanos desde una visión global.

\section{Conclusiones}

En primer lugar, vale la pena destacar que la evaluación realizada en el presente trabajo estuvo acotada a tres provincias con un alto grado de intercambio internacional —conforme a su desarrollo industrial y a sus estratégicos centros económicos-, lo que podría sesgar sus resultados a la hora de ser utilizados en otras provincias con menor grado de intercambio internacional. Además, la evaluación se realizó en 2008, conforme al primer plazo establecido por el RSI-2005 respecto al diagnóstico de las capacidades básicas en los Estados parte de la OMS. Su completa implementación, según el RSI-2005, debería concluirse en 2012, por lo que al día de hoy estos plazos están prácticamente vencidos.

Finalmente, se puede concluir que las provincias evaluadas cuentan con capacidades básicas instaladas que permitirán la implementación del RSI-2005 según el plazo previsto por la OMS. Es patente la necesidad de establecer y desarrollar planes de acción eficaces para consolidar a la vigilancia como una actividad esencial de seguridad nacional e internacional en términos de salud pública.

\section{Recomendaciones}

La inclusión del proceso de implementación del RSI-2005 en las estrategias del Estado y el proceso sistemático de planificación estratégica podrían garantizar el cumplimiento de los plazos estable- cidos y contribuir directamente con la salvaguarda de la seguridad nacional en términos de salud pública. En síntesis, se puede decir que para implementar acabadamente el RSI-2005 será de vital importancia tomar en consideración los siguientes puntos, a saber:

- Revisar y actualizar el marco legal nacional para que cuente con el respaldo jurídico necesario.

- Prestar especial atención a la recuperación y el fortalecimiento de la autoridad de inspección sanitaria estatal.

- Lograr una mayor vinculación entre las autoridades de los organismos nacionales a cargo del control sanitario y la actividad socioeconómica que realizan.

- Desarrollar tecnologías de soporte y estándares para la detección temprana, la evaluación de riesgo, la notificación y la coordinación de la respuesta frente a riesgos de salud pública.

- Fortalecer cada uno de los sistemas de vigilancia, articulados con el Centro Nacional de Enlace (CNE), para una efectiva comunicación, con un enfoque multisectorial y multidisciplinario.

- Desarrollar planes de emergencia, y actualizar los existentes, en todos los niveles del sistema sanitario y para cada tipo de amenaza en particular.

- Diseñar una estrategia de comunicación completa dirigida a todos los actores -incluidos los de la comunidad- para promover el conocimiento y aptitudes necesarios para enfrentarse a las emergencias de salud pública.

- Capacitar y mantener actualizado al personal de salud pública a través de una política sostenible de renovación continua de competencias.

Agradecimientos. Los autores desean agradecer a las direcciones nacionales de epidemiología, salud ambiental y relaciones internacionales del MINSAP, por todas las gestiones realizadas para llevar a cabo esta investigación, así como a la Organización Panamericana de la Salud, por su valioso apoyo técnico y asesoría. Asimismo, desean expresar su gratitud a la Escuela Latinoamericana de Salud Pública, por su invaluable ayuda en la validación del presente estudio, a todos los epidemiólogos, higienistas, médicos, administrativos, operadores y autoridades de las entidades evaluadas, y al Instituto Pedro Kourí, por el apoyo incondicional recibido a lo largo de toda la investigación. 


\section{REFERENCIAS}

1. World Health Organization. International Health Regulations (2005). 2. ${ }^{\text {a }}$ ed. Geneva: WHO; 2008.

2. Kamradt-Scott A. The evolving WHO: Implications for global health security. Glob Public Health. 2011;6(8):801-13.

3. Katz Rebecca L, Fernandez Jose A, McNabb SJN. Disease surveillance, capacity building and implementation of the International Health Regulations (IHR[2005]). BMC Public Health. 2010;10(1 supl):S1.

4. Armstrong KE, McNabb SJN, Ferland LD, Stephens T, Muldoon A, Fernandez JA, et al. Capacity of Public Health Surveillance to Comply with Revised International Health Regulations, USA. Emerg Infect Dis. 2010;16(5):804.

5. Taylor AL, McCarthy A, Haffajee R. Topic 01: International Health Regulations. In: Taylor AL, ed. An Overview of the IHR (2005); O’Neill Institute for National and Global Health Law. Geneva: WHO; 2010.

6. Hollmeyer H, Eckmanns T, Krause G. Surveillance under the International Health Regulations (2005). Bundesgesundheitsblatt Gesundheitsforschung Gesundheitsschutz. 2009;52(2):168-75.

7. Gala Gonzalez A. El reglamento sanitario internacional 2005: Aspectos importantes para su implementación. $1 .^{\text {a }}$ parte. La Habana: Instituto Pedro Kourí; 2011.

8. Gala Gonzalez A. El reglamento sanitario internacional 2005: Aspectos importantes para su implementación. 2. ${ }^{a}$ parte. La Habana: Instituto Pedro Kourí; 2011.
9. Wilson $\mathrm{K}, \mathrm{McD}$ ougall $\mathrm{C}$, Fidler DP, Lazar $\mathrm{H}$. Strategies for implementing the new International Health Regulations in federal countries. Bull World Health Organ. 2008;86(3):215-20.

10. Ministerio de Salud (MINSAL), Argentina. Caracterización de las capacidades básicas nacionales de vigilancia y respuesta. Informe del proceso y resultados. Buenos Aires: MINSAL; 2008.

11. Joseph F, Wamala J, Okot C, Makumbi I, Natseri N, Kisakye A, et al. Assessment of core capacities for the International Health Regulations (IHR[2005])—Uganda, 2009. BMC Public Health. 2010;10(1 supl):S9.

12. Alonso L, Pujadas M, Rosa R. Evaluación de capacidades básicas para cumplir el Reglamento Sanitario Internacional en puntos de entrada de Uruguay. Rev Panam Salud Publica. 2011;30(1):59-64.

13. Gala Gonzalez A. Reglamento Sanitario Internacional 2005: A propósito de la implementación. Rev Cubana de Salud Publica Internacional. 2011;2(1).

14. Donis R. Lessons Learned from the 2009 H1N1 Influenza Pandemic. Scientific Consultation On Influenza And Other Emerging Infectious Diseases At The Human-animal Interface. Verona: FAO/OIE/WHO; 2010.

15. World Health Organization. International Health Regulations (2005). Toolkit for implementation in national legislation. Questions and answers, legislative reference and assessment tool and examples of national legislation. Disponible en: http://www.who.int/
ihr/Toolkit_Legislative_Implementation.pdf Acceso el 8 de septiembre de 2012.

16. Universidad ISALUD. Marco de referencia para la implementación normativa del Reglamento Sanitario Internacional (2005). Disponible en: http://new.paho.org/hq/ dmdocuments/2010/Marco_de_Referencia RSI.pdf Acceso el 24 de agosto de 2012.

17. Aguilera $X$, Olea A, Muñoz X. Implementación del Reglamento Sanitario Internacional (2005). Reporte de progreso. Boletín de Vigilancia en Salud Pública. 2012;13(27):12-8.

18. Organización Panamericana de la Salud. Implementación del Reglamento Sanitario Internacional. Informe de progreso. Washington DC.: OPS; 2010.

19. Ministerio de Salud Pública (MINSAP), Cuba. Transformaciones necesarias en el sistema de salud pública. Disponible en: http://www. sld.cu/verpost.php?blog=http://articulos. sld.cu/editorhome $/$ \&post_id $=5186 \&$ tipo $=$ 1\&opc_mostrar=2_3_\&n=z Acceso el 8 de septiembre de 2012 .

20. Ministerio de Salud Pública (MINSAP), Cuba Plan para el enfrentamiento de la pandemia Influenza A (H1N1). La Habana, Cuba: MINSAP; 2009. Pp. 16-8.

Manuscrito recibido el 14 de junio de 2011. Aceptado para publicación, tras revisión, el 10 de julio de 2012.
ABSTRACT

\section{Implementation of the} International Health Regulations in Cuba: evaluation of basic capacities of the health sector in selected provinces

Key words
Objective. Obtain baseline information on the status of the basic capacities of the health sector at the local, municipal, and provincial levels in order to facilitate identification of priorities and guide public policies that aim to comply with the requirements and capacities established in Annex 1A of the International Health Regulations 2005 (IHR-2005).

Methods. A descriptive cross-sectional study was conducted by application of an instrument of evaluation of basic capacities referring to legal and institutional autonomy, the surveillance and research process, and the response to health emergencies in 36 entities involved in international sanitary control at the local, municipal, and provincial levels in the provinces of Havana, Cienfuegos, and Santiago de Cuba.

Results. The polyclinics and provincial centers of health and epidemiology in the three provinces had more than $75 \%$ of the basic capacities required. Twelve out of 36 units had implemented $50 \%$ of the legal and institutional framework. There was variable availability of routine surveillance and research, whereas the entities in Havana had more than $40 \%$ of the basic capacities in the area of events response.

Conclusions. The provinces evaluated have integrated the basic capacities that will allow implementation of IHR-2005 within the period established by the World Health Organization. It is necessary to develop and establish effective action plans to consolidate surveillance as an essential activity of national and international security in terms of public health.

International Health Regulations; evaluation; world health; international cooperation; Cuba. 